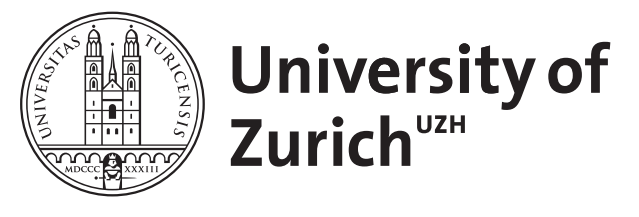

\title{
Biases in mass estimates of dSph galaxies
}

Lokas, E L ; Kazantzidis, S ; Klimentowski, J ; Mayer, L

\begin{abstract}
Using a high resolution N-body simulation of a two-component dwarf galaxy orbiting in the potential of the Milky Way, we study two effects that lead to significant biases in mass estimates of dwarf spheroidal galaxies. Both are due to the strong tidal interaction of initially disky dwarfs with their host. The tidal stripping of dwarf stars leads to the formation of strong tidal tails that are typically aligned with the line of sight of an observer positioned close to the host center. The stars from the tails contaminate the kinematic samples leading to a velocity dispersion profile increasing with the projected radius and resulting in an overestimate of mass. The tidal stirring of the dwarf leads to the morphological transformation of the initial stellar disk into a bar and then a spheroid. The distribution of stars in the dwarf remains non-spherical for a long time leading to an overestimate of its mass if it is observed along the major axis and an underestimate if it seen in the perpendicular direction.
\end{abstract}

DOI: https://doi.org/10.1063/1.3458535

Posted at the Zurich Open Repository and Archive, University of Zurich

ZORA URL: https://doi.org/10.5167/uzh-41702

Conference or Workshop Item

Accepted Version

Originally published at:

Lokas, E L; Kazantzidis, S; Klimentowski, J; Mayer, L (2009). Biases in mass estimates of dSph galaxies. In: Hunting for the Dark: the Hidden Side of Galaxy Formation, Qawra, Malta, 19 October 2009 - 23 October 2009. American Institute of Physics, 363-366.

DOI: https://doi.org/10.1063/1.3458535 


\title{
Biases in mass estimates of dSph galaxies
}

\author{
Ewa L. Łokas $^{1}$, Stelios Kazantzidis ${ }^{2}$, Jarosław Klimentowski ${ }^{1}$ and Lucio Mayer ${ }^{3}$
}

\begin{abstract}
Using a high resolution $N$-body simulation of a two-component dwarf galaxy orbiting in the potential of the Milky Way, we study two effects that lead to significant biases in mass estimates of dwarf spheroidal galaxies. Both are due to the strong tidal interaction of initially disky dwarfs with their host. The tidal stripping of dwarf stars leads to the formation of strong tidal tails that are typically aligned with the line of sight of an observer positioned close to the host center. The stars from the tails contaminate the kinematic samples leading to a velocity dispersion profile increasing with the projected radius and resulting in an overestimate of mass. The tidal stirring of the dwarf leads to the morphological transformation of the initial stellar disk into a bar and then a spheroid. The distribution of stars in the dwarf remains non-spherical for a long time leading to an overestimate of its mass if it is observed along the major axis and an underestimate if it seen in the perpendicular direction.
\end{abstract}

Subject headings: DSph galaxies, Tidal evolution, Mass estimates

\section{Introduction}

For the purpose of this study we used a high-resolution simulation of a two-component dwarf galaxy orbiting in the gravitational potential of the Milky Way (Klimentowski et al. 2007, 2009a). The dwarf progenitor of total mass $M=4.3 \times 10^{9} M_{\odot}$ consisted of a baryonic disk embedded in a dark matter halo. The initial mass of the disk was $1.5 \times 10^{8} M_{\odot}$ and the mass of the NFW dark matter halo was $4.1 \times 10^{9} M_{\odot}$. The dwarf galaxy was placed on an eccentric orbit around the host galaxy with apocenter to pericenter ratio of $r_{\mathrm{a}} / r_{\mathrm{p}}=120 / 25 \mathrm{kpc}$ and the disk initially inclined by $45^{\circ}$ to the orbital plane. The evolution was followed for $10 \mathrm{Gyr}$ corresponding to five orbital times. The host galaxy was modelled by a static gravitational potential assumed to have the present-day properties of the Milky Way as described by mass model A1 of Klypin et al. (2002).

During the evolution the dwarf galaxy is strongly affected by the tidal field of the host which results in a significant mass loss, the morphological transformation from a disk to a bar and then

\footnotetext{
${ }^{1}$ Nicolaus Copernicus Astronomical Center, 00-716 Warsaw, Poland; lokas@camk.edu.pl

${ }^{2}$ Center for Cosmology and Astro-Particle Physics, The Ohio State University, Columbus, OH 43210, USA; stelios@mps.ohio-state.edu

${ }^{3}$ Institute for Theoretical Physics, University of Zürich, CH-8057 Zürich, Switzerland; lucio@phys.ethz.ch
} 

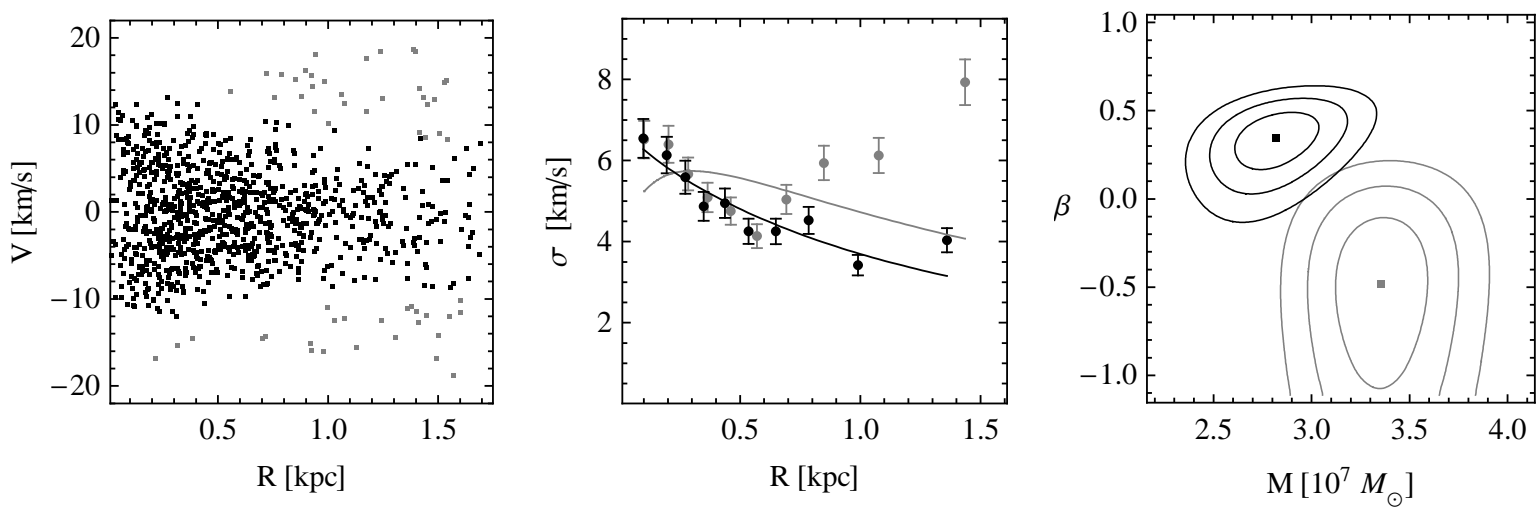

Fig. 1. - An example of a kinematic sample of a thousand stars (left panel), the velocity dispersion profiles measured from it (middle panel) and the contours showing the inferred mass and anisotropy (right panel). In each panel the gray (black) color refers to the sample contaminated by (cleaned of) interloper stars from the tails.

a spheroid and the transition from the streaming to the random motion of the stars. At all times, including the final stage, the core of the dwarf galaxy remains gravitationally bound but is surrounded by pronounced tidal tails and its shape departs from spherical. If dwarf spheroidal galaxies of the Local Group indeed formed as envisioned by this so-called tidal stirring scenario (Mayer et al. 2001, 2007), these effects must be taken into account when modeling their masses. Here we provide quantitative estimates of the biases imposed by the contamination of kinematic samples by unbound stars from the tails and by the departures from sphericity of the stellar component.

\section{Contamination of kinematic samples by tidally stripped stars}

The left panel of Fig. 1 shows a kinematic sample of a thousand stars randomly selected from the final output of the simulation (where the dwarf galaxy is at apocenter). This mock data set shows line-of-sight velocities as a function of the projected distance $R$ as would be measured by a distant observer. The observation is made along the tidal tails, which is a typical orientation of the debris for an observer placed near the center of the Milky Way (Klimentowski et al. 2009b). Only the data within the standard velocity cut-off of $\pm 3 \sigma_{0}$ (where $\sigma_{0}$ is the central velocity dispersion of the stars) from the systemic velocity of the dwarf are shown. From this data set we obtain the velocity dispersion profile shown in the middle panel of Fig. 1 with gray data points with sampling errors. We then fit to this profile the solutions of the Jeans equation for spherical systems, assuming that mass traces light (the distribution of stars is measured for the same line of sight), and adjusting two parameters: the total mass $M$ and the anisotropy parameter of stellar orbits $\beta=1-\sigma_{\theta}^{2} / \sigma_{r}^{2}$. 

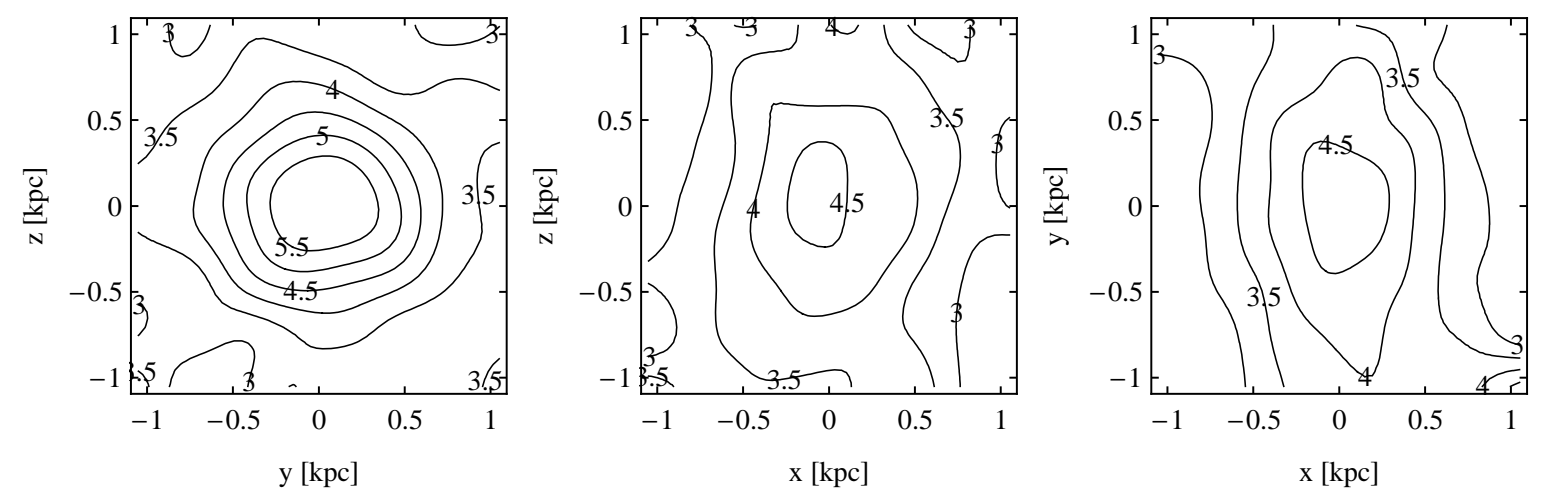

Fig. 2.- Maps of the line-of-sight velocity dispersion of the stars measured along the long (left panel) and the two short axes (middle and right panel). Numbers give the values of the velocity dispersion in $\mathrm{km} \mathrm{s}^{-1}$.

The best-fitting solution is shown as a gray line in the middle panel of Fig. 1 and the constraints on $M$ and $\beta$ are shown with the gray $1 \sigma, 2 \sigma$ and $3 \sigma$ contours in the right panel of Fig. 1.

The increase of the velocity dispersion profile for $R>0.7 \mathrm{kpc}$ is due to the contamination of the sample by unbound stars from the tidal tails. Note that the true values of the mass and anisotropy of this dwarf galaxy within a sphere of $r<1.7 \mathrm{kpc}$ measured in the simulation are respectively $M=2.1 \times 10^{7} M_{\odot}$ and $\beta=0.33$ (Eokas et al. 2010). The use of the contaminated sample thus leads to an overestimate of the mass and an underestimate of the anisotropy (tangential rather than mildly radial orbits are inferred). The amount of this bias will depend on the actual orientation of the tails and the initial velocity cut-off applied to the sample, but the presented example shows that it can be quite large. Since the fit is rather bad one may try to improve it by introducing a more extended dark matter halo. The tidal stirring scenario predicts, however, that tidally affected dwarfs do not possess such halos and their mass approximately traces light (Mayer et al. 2001, Łokas et al. 2010).

The problem is solved when the kinematic sample is cleaned of interlopers (Klimentowski et al. 2007, Łokas et al. 2008, Łokas 2009). The stars marked by the gray dots in the left panel of Fig. 1 have been removed by a scheme based on assigning a maximum velocity allowed for a star at a given $R$ which was extensively tested on simulated data (Klimentowski et al. 2007). The resulting velocity dispersion profile (black points in the middle panel of Fig. 1) is then no longer increasing with radius and our simple two-parameter model fits the data much better. The best-fitting mass and anisotropy (black contours in the right panel of Fig. 1) obtained in this case are in very good agreement with the real values. 

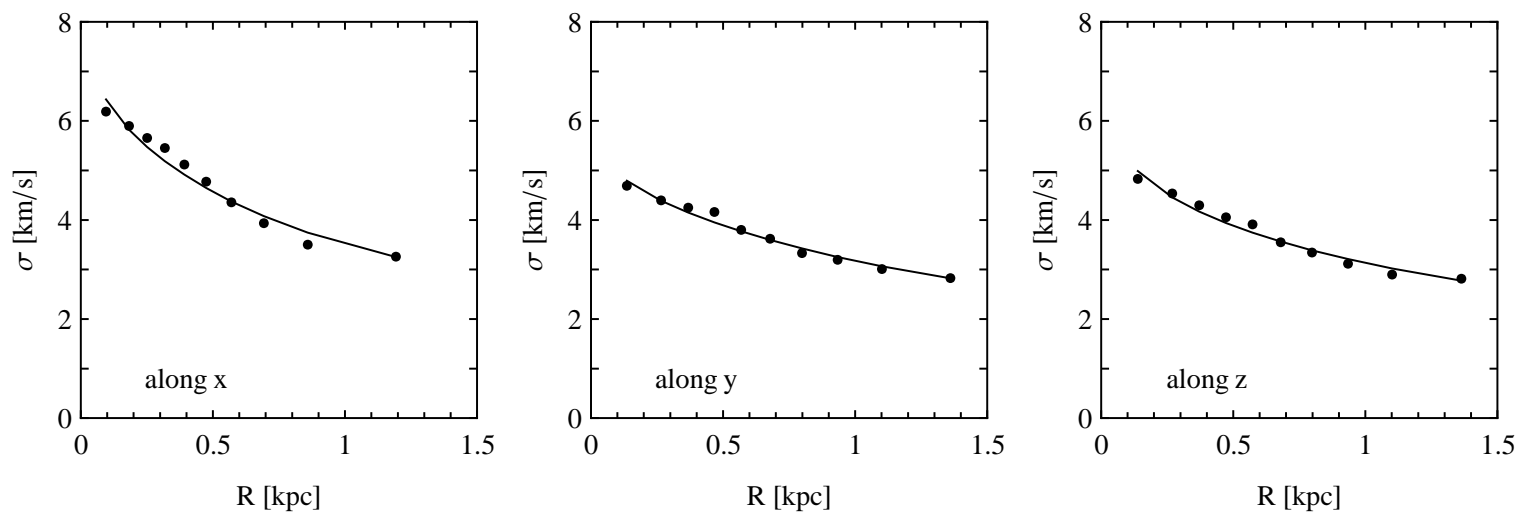

Fig. 3.- Line-of-sight velocity dispersion profiles of the stars when the dwarf is observed along the long (left panel) and one of the two short axes (middle and right panel). The solid lines show the best-fitting solutions of the Jeans equation.

\section{The effect of non-sphericity of the dwarf}

The products of tidal stirring are typically non-spherical. In the example discussed here the distribution of the stars in the final stage is a prolate spheroid with the short-to-long axis ratio of 0.6 (Klimentowski et al. 2009a). The shape affects the velocity distribution in the dwarf which retains some of the radial orbits characteristic of the long-lasting bar-like phase of the evolution where the axis ratio was much smaller. Fig. 2 shows the maps of the line-of-sight velocity dispersion of the stars seen along the long $(x)$ and the two short ( $y$ and $z$ ) axes (from the left to the right panel). The dispersion is larger when viewed along the $x$ axis and it decreases more steeply with radius.

The same effect is seen in Fig. 3 where we plot the velocity dispersion profiles measured in a traditional way, in concentric shells of projected radius $R$ : when measured along the long axis of the stellar component the dispersion reaches higher values in the center and decreases more steeply with $R$. Note that here only the stars within the sphere of radius $r<1.7 \mathrm{kpc}$ were used so no contamination from the tidal tails is present and all profiles decrease with radius. In addition, we used all (i.e. almost 38 thousand) stars, rather than a sample of a thousand to avoid the noise due to sampling errors (those are then too small to be visible in Fig. 3 and are not shown).

As in the previous section, we fitted the dispersion profiles with the solutions of the Jeans equation for spherical systems, adjusting the total mass and anisotropy, assuming that mass follows light (the stellar density profile was fitted separately for every line of sight). For the view along $x$ the mass within $1.7 \mathrm{kpc}$ is overestimated by $7 \%$, for the view along $y$ and $z$ it is underestimated by $17-18 \%$. Interestingly, the anisotropy is recovered almost perfectly, with the best-fitting values within the range of $\beta=0.2-0.3$ in all cases (Eokas et al. 2010). 
This research was partially supported by the Polish Ministry of Science and Higher Education under grant NN203025333.

\section{REFERENCES}

J. Klimentowski, E. L. Łokas, S. Kazantzidis, F. Prada, L. Mayer, G. A. Mamon, MNRAS 378, 353-368 (2007).

J. Klimentowski, E. L. Łokas, S. Kazantzidis, L. Mayer, G. A. Mamon, MNRAS 397, 2015-2029 (2009).

A. Klypin, H. Zhao, R. S. Somerville, ApJ 573, 597-613 (2002).

L. Mayer, F. Governato, M. Colpi, B. Moore, T. Quinn, J. Wadsley, J. Stadel, G. Lake, ApJ 559, 754-784 (2001).

L. Mayer, S. Kazantzidis, C. Mastropietro, J. Wadsley, Nature 445, 738-740 (2007).

J. Klimentowski, E. L. Łokas, S. Kazantzidis, L. Mayer, G. A. Mamon, F. Prada, MNRAS 400, $2162-2168$ (2009).

E. L. Łokas, S. Kazantzidis, J. Klimentowski, L. Mayer, S. Callegari, ApJ 708, 1032-1047 (2010).

E. L. Łokas, J. Klimentowski, S. Kazantzidis, L. Mayer, MNRAS 390, 625-634 (2008).

E. L. Lokas, MNRAS 394, L102-L106 (2009). 